\title{
Cinética de absorção com doses de fósforo e fungos micorrízicos arbusculares em Nicotiana tabacum
}

\section{P uptake kinetics in Nicotiana tabacum plants subjected to doses of phosphorus and arbuscular mycorrhizal fungi}

\author{
José Ozinaldo Alves de SENA ${ }^{1 ; 2}$; Ronaldo STEFANUTTI ${ }^{3}$; Riviane Maria Albuquerque DONHA ${ }^{4}$; \\ Elke Jurandy Bran Nogueira CARDOSO ${ }^{5}$ \\ ${ }^{1}$ Trabalho complementar da tese de doutorado do primeiro autor; Pesquisa financiada pela CAPES \\ 2 Doutor;Universidade Estadual de Maringá;ozisena@hotmail.com \\ ${ }^{3}$ Doutor; Universidade Federal do Ceará ; ronaldostefanutti@hotmail.com \\ ${ }^{4}$ Mestranda; Centro de Energia Nuclear na Agricultura; Biologia na Agricultura e Ambiente, Nutrição Mineral de \\ Plantas; Av. Centenário, 303; rmdonha@cena.usp.br \\ ${ }^{5}$ Doutora, Escola Superior de Agricultura 'Luiz de Queiroz', ejbncard@esalq.usp.br
}

Recebido em: 14-06-2013; Aceito em: 17-04-2014

\begin{abstract}
Resumo
Do ponto de vista nutricional, as micorrizas arbusculares estão envolvidas em mecanismos que aumentam a absorção de nutrientes, como o fósforo. Com o objetivo de se estudar a cinética de absorção de $\mathrm{P}$ por plantas de tabaco micorrizadas, instalou-se experimento em casa de vegetação usando Nicotiana tabacum como planta-teste, sem inoculação (controle) e com espécies de fungos micorrízicos arbusculares (Glomus intraradices e Gigaspora margarita), além de duas doses de P (40 e $120 \mathrm{mg} \mathrm{kg}^{-1}$ ) e $\mathrm{o}$ tratamento-controle. Para o estudo de cinética de absorção, foram usadas raízes destacadas e quatro concentrações externas de $\mathrm{P}$, em mol L-1 $\left(2.10^{-2} ; 2.10^{-4} ; 2.10^{-6}\right.$ e $\left.2.10^{-8}\right)$. As soluções obtidas por digestão nitroperclórica foram levadas para contador de cintilação líquida, e as contagens, ao final, expressas em $\mu \mathrm{mol}$ de ${ }^{32} \mathrm{P}$ por grama de matéria seca de raiz e por 120 minutos. Os sistemas micorrízicos foram influenciados pela adubação fosfatada, pois o $\mathrm{Km}$ e Vmáx. sofreram alterações, variando a intensidade deste efeito em função da espécie de FMA. Plantas colonizadas com G.intraradices mostraram-se com maior capacidade de absorção de $\mathrm{P}$ em condições de baixa disponibilidade, porém menos responsivas ao incremento do elemento no meio.
\end{abstract}

Palavras-chave adicionais: cinética de absorção iônica; ${ }^{32} \mathrm{P}$; Gigaspora margarita; Glomus intraradices.

\begin{abstract}
From a nutritional point-of-view, the arbuscular mycorrhiza are involved in mechanisms that increase nutrients absorption, such as phosphorus. With the aim of studying the kinetics of $\mathrm{P}$ uptake by mycorrhizal tobacco plants, a greenhouse experiment was set using as a test plants of Nicotiana tabacum, uninoculated (control) and inoculated with arbuscular mycorrhizal fungi (Glomus intraradices and Gigaspora margarita) in addition to two doses of $\mathrm{P}$ (40 and $120 \mathrm{mg} \mathrm{kg}^{-1}$ ) and the control treatment. For the study of the absorption kinetics excised roots and four external concentration of $P$, in $\mathrm{mol}^{-1}$ $\left(2.10^{-2} ; 2.10^{-4} ; 2.10^{-6} \mathrm{e} 2.10^{-8}\right)$. The solutions resulting from the nitroperchloric digestion were taken to a liquid scintillation counter which were expressed in $\mu$ mols of ${ }^{32} \mathrm{P} / \mathrm{gram}$ of root dry matter for 120 minutes. The mycorrhizal systems were influenced by the phosphorous fertilization since $\mathrm{Km}$ and Vmax underwent alterations, the intensity of which depended on the FMA species. Plants colonized by $G$. intraradices showed a higher P absorption capacity when this element is in low availability in the soil although less responsive to the increment of that element in the medium.
\end{abstract}

Additional keywords: Gigaspora margarita; Glomus intraradices; ion absorption kinetics; ${ }^{32} \mathrm{P}$.

\section{Introdução}

As atividades de cultivo do fumo estão dispersas em mais de 100 países em todo o mundo, o Brasil tornou-se recentemente o segundo maior produtor mundial e consolidou sua posição como o maior exportador mundial da cultura (VARGAS ;OLIVEIRA, 2012). Seu cultivo é realizado de modo convencional, sem práticas conservacionistas e com usos intensivos de pesticidas e fertilizantes (GONÇALVES et al., 2005).

Nas condições do País, a produtividade de diversas culturas é frequentemente limitada pela deficiência de $\mathrm{P}$ no solo. Os teores desse elemento são geralmente muito baixos, sendo necessárias adubações relativamente pesadas para aumentar o $\mathrm{P}$ disponível às culturas. $\mathrm{O}$ fósforo é um elemento de baixo aproveitamento na 
agricultura, o conhecimento das habilidades das plantas em absorver o nutriente do solo e utilizálo em seu crescimento é uma alternativa que pode ser importante para melhorar sensivelmente a eficiência da adubação fosfatada (LUCA et al., 2002).

O desenvolvimento de variedades eficientes em $\mathrm{P}$ pode obter melhor produção com suprimento baixo de $\mathrm{P}$, sendo chave para melhorar a produção agrícola. Em condições deficientes de $P$, as plantas desenvolveram uma série de estratégias adaptativas para ocupar e utilizar o solo com P (ZHANG et al., 2009). Na aquisição de $P$, as características mais importantes são as características morfológicas da raiz e da arquitetura (WANG et al., 2010). A diferença na seleção dos genótipos quanto à eficiência de uso de $\mathrm{P}$ e à produção de matéria seca está relacionada com a translocação do $\mathrm{P}$ das raízes para a parte aérea (LANA et al., 2006).

Contudo, são as associações com fungos micorrízicos arbusculares (FMA) o fator primordial para o aumento da absorção de $P$ pelas plantas, sobretudo em ambientes pobres em $P$ (CHAVES DE OLIVEIRA \& REIS DE CARVALHO, 2011). O estudo de FMA é uma estratégia com o objetivo de beneficiar a nutrição das plantas e sua produtividade com menores investimentos com insumos e maior sustentabilidade no sistema.

Diferenças inter e intraespecíficas na eficiência de utilização de fósforo no solo $(P)$ são explicadas, em parte, através de variações morfológicas e fisiológicas das plantas, que caracterizam a planta para a aquisição de nutrientes. $A$ cinética de absorção de $P$ e a capacidade de plantas na manutenção de um nível metabólico normal dos tecidos com concentrações mais baixas de $P$ são mecanismos fisiológicos que lidam com plantas para atingir maior eficiência de utilização de P (MACHADO et al., 2004).

A variação dos parâmetros cinéticos de absorção Km (constante de Michaelis-Menten), Vmax. (velocidade máxima) e Cmín. (concentração mínima) são amplas e estão relacionadas à morfologia, fisiologia e desenvolvimento do sistema radicular, o que significa indicativo de diferenças genotípicas quanto à capacidade de adaptação aos diversos ecossistemas (BAPTISTA et al., 2000). A marcha de absorção de nutrientes é referência importante para o fornecimento dos mesmos em doses adequadas para o desenvolvimento das plantas (BARBOSA et al., 2003). Os valores dos parâmetros cinéticos de absorção variaram com os genótipos e com os nutrientes (HORN et al., 2006).

Visando a contribuir para o entendimento dos mecanismos relacionados à eficiência no aproveitamento de $\mathrm{P}$ por plantas micorrizadas, bem como a gerar informações que sejam úteis a estudos envolvendo fisiologia e melhoramento de plantas, que o presente experimento foi realizado.

\section{Material e métodos}

O experimento foi desenvolvido em casa de vegetação e laboratórios, comparando-se a absorção iônica de $\mathrm{P}$, por raízes destacadas de tabaco (Nicotiana tabacum), oriundas de plantas cultivadas em vasos, com substrato composto por uma mistura de areia lavada e vermiculita, na proporção de $3: 1$, onde se adicionaram as doses de $\mathrm{P}$ a serem estudadas, equivalentes a 0,40 e $120 \mathrm{mg} \mathrm{kg}^{-1}$.

Foi feita inoculação com os fungos micorrízicos arbusculares (FMA) Glomus intraradices ou Gigaspora margarita, além da planta-testemunha sem inoculante. Cem dias após o transplantio, as mudas foram retiradas dos vasos, lavadas em água corrente e água destilada, e colocadas em solução de Hoagland ; Arnon (1950) modificada (1/10 dos macronutrientes - $P$ e $1 / 2$ dos micronutrientes) por 24 horas, sob constante aeração (FAQUIN, 1988).

Após esse período, logo abaixo do colo, lavadas em água destilada e colocadas em frascos de $200 \mathrm{~mL}$, contendo $100 \mathrm{~mL}$ de solução de $\mathrm{NaH}_{2}{ }^{32} \mathrm{PO}_{4}$ nas concentrações $2.10^{-2} \mathrm{~mol} \mathrm{~L}^{-1}$, $2.10^{-4} \mathrm{~mol} \mathrm{~L}^{-1}, 2 \cdot 10^{-6} \mathrm{~L}^{-1}, 2.10^{-8} \mathrm{~mol} \mathrm{~L}^{-1}$ de $\mathrm{P}$. Foram adicionados em todos os tratamentos $1 \mathrm{~mL}$ de $\mathrm{MgSO}_{4} 2 \cdot 10^{-2} \mathrm{~mol} \mathrm{~L}^{-1} 2 \cdot 10^{-2} \mathrm{~mol} \mathrm{~L}^{-1}$ e $1 \mathrm{~mL}$ de $\mathrm{NaH}_{2}{ }^{32} \mathrm{PO}_{4}$ livre de carreador. Os tratamentos foram conduzidos em três repetições, mantendose as raízes em solução, sob constante aeração, por 120 minutos (MALAVOLTA et al., 1992).

Decorrido este tempo, as raízes foram retiradas e lavadas com água destilada, agitando-se os frascos durante 1 minuto, três vezes seguida. As raízes foram secas em estufa de circulação forçada de ar a $65^{\circ} \mathrm{C}$, até peso constante. Foi determinada a massa e, em seguida, as raízes foram submetidas à digestão nitricoperclórica (MALAVOLTA et al., 1989).

As contagens (cpm) foram convertidas em $\mu \mathrm{mol}$ de ${ }^{32} \mathrm{P}$ por grama de matéria seca de raiz, por 120 minutos, segundo HARRISON \& HELLIWELL (1979). Para interpretação do processo de absorção por raízes destacadas, calcularam-se os valores de $\mathrm{Km}$ e Vmáx. com a equação de HOFSTEE (1952), que apresentou o melhor ajuste.

\section{Resultados e discussão}

Os sistemas micorrízicos foram influenciados pela adubação fosfatada, pois os valores de $\mathrm{Km}$ e Vmáx. sofreram alterações, variando a intensidade deste efeito em função da espécie de fungo micorrízico (Tabela 1 ). Em relação aos resultados de $P$ na planta, os resultados correspondem com os trabalhos em literatura, descritos 
por SILVEIRA \& CARDOSO (1990), CUNHA (1999) e SILVEIRA et al., (2004). Em geral, as plantas micorrizadas apresentaram maior $\mathrm{V}$ máx. e menores valores de $\mathrm{Km}$, com as plantas na presença de fungos micorrízicos demonstrando maior influxo de $P$ quando comparadas às não micorrizadas, em condições ambientais semeIhantes (SILVEIRA et al, 2004). Os menores valores de $\mathrm{Km}$ são bons indicadores da capacidade de absorção de $\mathrm{P}$.

Tabela 1. Valores dos parâmetros Km e Vmáx. determinados com as transformações de HOFSTEE (1952), referentes à absorção de $\mathrm{P}$ por raízes destacadas de tabaco, nas concentrações de $2.10^{-2} \mathrm{~mol} \mathrm{~L}^{-1}, 2 \cdot 10^{-4} \mathrm{~mol} \mathrm{~L}^{-1}, 2 \cdot 10^{-6} \mathrm{~mol} \mathrm{~L}^{-1}, 2 \cdot 10^{-8} \mathrm{~mol} \mathrm{~L}^{-1} \mathrm{de} \mathrm{NaH}_{2}{ }^{32} \mathrm{PO}_{4}$. Values of the parameters $\mathrm{Km}$ and Vmax determined by the HOFSTEE(1952) transformations, related to $P$ uptake by excised roots of tobacco, at concentrations of $2.10^{-2} \mathrm{~mol} \mathrm{~L}^{-1}, 2.10^{-4} \mathrm{~mol} \mathrm{~L}^{-1}, 2 \cdot 10^{-6} \mathrm{~mol} \mathrm{~L}^{-1}, 2.10^{-8} \mathrm{~mol} \mathrm{~L}^{-1} \mathrm{de} \mathrm{NaH}_{2}^{32} \mathrm{PO}_{4}$.

\begin{tabular}{|c|c|c|c|}
\hline $\begin{array}{c}\text { Tratamentos } \\
\left(\mathrm{mg} \mathrm{kg}^{-1}\right)\end{array}$ & Equações & $\begin{array}{c}\mathrm{Km} \\
\left(\mu \mathrm{mol} \mathrm{L}^{-1}\right)\end{array}$ & $\begin{array}{c}\text { Vmáx. } \\
\left(\mu \mathrm{mol} \mathrm{g}{ }^{-1} 2 h^{-1}\right)\end{array}$ \\
\hline (a) Controle & $Y=0,39411+0,00663 x$ & 59,5 & 15,9 \\
\hline (b) $40 \mathrm{c} / \mathrm{FMA}-\mathrm{Gl}$ & $Y=1,17405+0,00682 x$ & 17,2 & 14,7 \\
\hline (c) $40 \mathrm{c} / \mathrm{FMA}$ - GM & $Y=1,45378+0,03055 x$ & 47,2 & 32,7 \\
\hline $40 \mathrm{~s} / \mathrm{FMA}$ & $Y=1,30851+0,04281 x$ & 30,6 & 23,4 \\
\hline 120 c/ FMA - GI & $Y=2,488+0,5462 x$ & 45,5 & 18,3 \\
\hline $120 \mathrm{c} / \mathrm{FMA}-\mathrm{GM}$ & $Y=3,31446+0,00617 x$ & 53,2 & 16,1 \\
\hline 120 s/ FMA & $Y=1,87285+0,0399 x$ & 46,7 & 25,1 \\
\hline
\end{tabular}

Em estudos de cinética de absorção, usando-se de raízes destacadas, raízes de plantas micorrizadas tendem a apresentar maior afinidade para o fosfato (menor $\mathrm{Km}$ ), em comparação com o controle não micorrizado, não sendo afetada, por outro lado, a taxa máxima de absorção (Vmáx.) (CRESS et al., 1979). Resultados contrários a estes também têm sido observados em trabalhos da literatura, com valores mais altos de Vmáx. para raízes de plantas micorrizadas (aumento no número de sítios de absorção resultantes da presença do fungo) (FAQUIN et al., 1990) ou maior Vmáx. e menor $\mathrm{Km}$ (SILVEIRA \& CARDOSO, 1990). Os resultados, portanto, podem depender da espécie do fungo micorrízico, da espécie de planta e do estádio de desenvolvimento em que a mesma se encontra, da colonização radicular, dentre outros.

Nas doses de 40 e $120 \mathrm{mg} \mathrm{kg}^{-1}$, verificaram-se valores de $\mathrm{Km}$ inferiores para raízes colonizadas por Glomus intraradices, em relação a Gigaspora margarita, indicando que as plantas de tabaco colonizadas por aquela espécie tenderam a apresentar maior capacidade de absorção de fósforo em condições de baixa concentração do elemento. Trabalhos semelhantes são encontrados em literatura, com dose de fósforo adequada em associação com Glomus sp. sendo indicadas para o crescimento de plantas de urucum, por promoverem adequadas respostas dos índices fisiológicos, contribuindo com o desenvolvimento (BARBIERI et al., 2011). A capacidade em promover o crescimento da planta pode variar em razão da planta, do ambiente e das espécies de fungos que formam micorrizas arbusculares, podendo diferir na eficiência em aumentar a absorção de $\mathrm{P}$ e favorecer $\mathrm{o}$ crescimento da planta (CAPRONI et al., 2003).
Esse efeito de maior afinidade para Glomus intraradices decresce com o aumento da dose de fosfato. Com o aumento da dose de fosfato de 40 para $120 \mathrm{mg} \mathrm{kg}^{-1}$, verificaram-se aumentos de $165 \%$ e $1.029 \%$ no $\mathrm{Km}$, para as raízes colonizadas com Glomus intraradices e Gigaspora margarita, respectivamente. O Vmáx. apresentou valores inferiores em plantas colonizadas com Glomus intraradices, apresentando pequena variação com o aumento da dose de fosfato, não sendo o mesmo observado com Gigaspora margarita, em que os valores de Vmáx. apresentaram grande aumento quando a dose de fosfato variou de 40 para $120 \mathrm{mg} \mathrm{kg}^{-1}$ (396\%), indicando que as raízes de plantas colonizadas com Gigaspora margarita podem ser responsivas ao aumento de $P$.

A análise da compatibilidade fungo-hospedeiro e o comportamento geral das espécies em associação indicam a ocorrência de seletividade diferenciada na relação fungo-hospedeiro, existindo combinações de eficiência simbiótica muito variável para as plantas hospedeiras (POUYU-ROJAS et al., 2006)

As raízes de plantas colonizadas com Glomus intraradices mostraram-se mais eficientes na absorção de $\mathrm{P}$ em condições de baixa disponibilidade $\left(40 \mathrm{mg} \mathrm{kg}^{-1}\right)$, porém são menos responsivas ao incremento de $P$ no meio. Em relação ao sistema sem aplicação de $P$, a presença de fungo micorrízico arbuscular aumentou a afinidade das raízes a este nutriente, principalmente para Glomus intraradices e com exceção de Gigaspora margarita, na dose de $120 \mathrm{mg} \mathrm{kg}^{-1}$. Estudos em literatura mostram que doses baixas de $\mathrm{P}$ aumentam a micorrização e a eficiência dos fungos micorrízicos em promover 
aumento na matéria seca; entretanto, altas doses desse nutriente afetam negativamente a micorrização (BRESSAN et al., 2001).

\section{Conclusões}

Os sistemas micorrízicos estudados foram influenciados pela adubação fosfatada, pois os parâmetros cinéticos $\mathrm{Km}$ e Vmáx. sofreram significativas alterações.

Plantas de tabaco colonizadas com Glomus intraradices apresentaram maior afinidade ao elemento $P$ (menor $\mathrm{Km}$ ), em condições de baixa concentração do elemento.

Plantas de tabaco colonizadas com Gigaspora margarita mostraram-se mais responsivas ao aumento de $\mathrm{P}$.

\section{Referências}

BAPTISTA, J. A.; FERNANDES, M. S.; SOUZA, S. R. Cinética de absorção de Amônio e crescimento radicular das cultivares de arroz Agulha e Bico Ganga. Pesquisa Agropecuária Brasileira, Brasília, v.35, n.7, p.1325-1330, 2000.

BARBIERI, D. J; BRAGA, L. F; SOUSA, M. P.; ROQUE, C. G. Análise de crescimento de Bixa orellana $L$. sob efeito da inoculação micorrízica e adubação fosfatada. Revista Brasileira de Plantas Medicinais, Botucatu, v.13, n.2, p.129-138, 2011.

BARBOSA, Z. ; SOARES, I. ; CRISOSTOMO, L. A. Crescimento e absorção de nutrientes por mudas de gravioleira. Revista Brasileira Fruticultura, Jaboticabal, v.25, n.3, p.519-522, 2003.

BRESSAN, W.; SIQUEIRA, J. O.; VASCONCELLOS, C. A.; PURCINO, A. A. C. Fungos micorrízicos e fósforo, no crescimento, nos teores de nutrientes e na produção do sorgo e soja consorciados. Pesquisa Agropecuária Brasileira, Brasília, v.36, n.2, p.315323, 2001.

CAPRONI, A. L.; FRANCO, A. A.; BERBARA, R. L. L.; TRUFEM, S. B.; GRANHA, J. R. D. O.; MONTEIRO, A. B. Ocorrência de fungos micorrízicos arbusculares em áreas revegetadas após mineração de bauxita em Porto Trombetas, Pará. Pesquisa Agropecuária Brasileira, Brasília, v.38, n.12, p.937-945, 2003.

CHAVES DE OLIVEIRA, P.; REIS DE CARVALHO, J. C. Rizosferas de árvores acumuladoras de fósforo na Amazônia Brasileira. Universitas Scientiarum, Bogotá, v.16, n.2, p.111-118, 2011.

CRESS, W. A.; THRONEBERRY, G. O.; LINDSEY; D. L. Kinetics of phosphorus absorption by mycorrhizal and nonmycorrhizal tomato roots. Plant Physiology, Minneapolis, v.64, n.3, p.484-487, 1979.
CUNHA, M. I. B. Parasitismo de fungos micorrízicos arbusculares e cinética de absorção de fósforo em plantas cítricas. 1999. $106 \mathrm{f}$. Dissertação (Mestrado) - Escola Superior de Agricultura "Luiz de Queiroz". Universidade de São Paulo, Piracicaba, 1999.

FAQUIN, V. Cinética de absorção de fosfato, nutrição mineral, crescimento e produção da soja sob influência de micorriza vesiculoarbuscular (MVA).1998. 136f. Tese (Doutorado) Escola Superior de Agricultura "Luiz de Queiroz". Universidade de São Paulo, Piracicaba, 1998.

GONÇALVES, C. S.; RHEINHEIMER, D. S.; PELLEGRINI, J. B. R.; KIST, S. L. Qualidade da água numa microbacia hidrográfica de cabeceira situada em região produtora de fumo. Revista Brasileira de Engenharia Agrícola e Ambiental, Campina Grande, v.9, n.3, p.391-399, 2005.

HARRISON, A. F.; HELLIWELL, D. R. A bioassay for comparing phosphorus availability in soils. Journal of Applied Ecology, Oxford, v.16, n.2, p.467-505, 1979.

HOAGLAND, D; ARNON, D. I. The water culture method for growing plants without soil. California Agriculture Experimental Station Circular, 1950. 347p.

HOFSTEE, B. H. J. On the evaluation of constants $\mathrm{Vm}$ and $\mathrm{Km}$ in enzyme reactions. Science, Washington, v.16, n.3013, p.329-333, 1952.

LANA, R. M. Q.; ZANAO JUNIOR, L. A.; CORREIA, N. M.; LANA, Â. M. Q. Variabilidade entre genótipos de feijoeiro na eficiência no uso do fósforo. Ciência Rural, Santa Maria, v.36, n.3, p.778-784, 2006.

LUCA, E. F.; BOARETTO, A. E.; MURAOKA, T.; CHITOLINA, J. C. Eficiência de absorção e utilização de fósforo (32P) por mudas de eucalipto e arroz. Scientia Agrícola, Piracicaba, v.59, n.3, p.543-547, 2002.

MACHADO, C. T. T.; FURLANI, A. M. C. Cinética de absorção de fósforo e morfologia radicular de variedades locais e melhoradas de milho. Scientia Agrícola, Piracicaba, v.61, n.1, p.69-76, 2004.

MALAVOLTA, E. ABC da análise de solos e folhas: amostragem, interpretação e sugestões de adubação. São Paulo: Agrônomica Ceres, 1992. 124p.

MALAVOLTA, E. ; VITTI, G. C.; OLIVEIRA, S. A. de. Avaliação do estado nutricional das plantas: princípios e aplicações. Piracicaba: POTAFOS, 1989. 201p. 
POUYU-ROJAS, E. ; SIQUEIRA, J. O.; SANTOS, J. G. D. Compatibilidade simbiótica de fungos micorrízicos arbusculares com espécies arbóreas tropicais. Revista Brasileira de Ciência do Solo,Viçosa, v.30, n.3,p.413-424, 2006.

SILVEIRA, A. P. D. CARDOSO, E. J. B. N. Kinetics of phosphorus uptake, growth and mineral nutrition of mycorrhizal and non-mycorrhizal bean (Phaseolus vulgaris L.). In: NORTH AMERICAN CONFERENCE ON MYCORRHIZAE, 8, 1990, Wyoming. Abstracts... Wyoming, 1990. p.269.

SILVEIRA, R. L. V. A.; HIGASHI, E. N.; GONÇALVES, A. N.; MOREIRA, A. Evaluation of the nutritional status of eucalypts: visual and foliar diagnoses and their interpretation. In: GONÇALVES, J. L. M.; BENEDETTI, V. (Ed). Forest nutrition and fertilization. Piracicaba: IPEF, 2004. p.79-104.
VARGAS, M. A.; OLIVEIRA, B. F. Estratégias de diversificação em áreas de cultivo de tabaco no Vale do Rio Pardo: uma análise comparativa. Revista de Economia e Sociologia Rural, Brasília, v.50, n.1, p.157-174, 2012.

WANG, X.; SHEN, J.; LIAO, H. Acquisition or utilization, which is more critical for enhancing phosphorus efficiency in modern crops? Plant Science, Limerick, v.179, n.4, p.302-306, 2010.

ZHANG, H.; HUANG, Y.; YE, X.; SHI, L.; XU, F. Genotypic differences in phosphorus acquisition and the rhizosphere properties of Brassica napus in response to low phosphorus stress. Plant Soil, The Hague, v.320, n.1, p.91-102, 2009. 